\title{
Title:
}

Comparison of the $\left[{ }^{18} \mathrm{~F}\right]$-FDG and $\left[{ }^{18} \mathrm{~F}\right]$-FLT PET Tracers in the Evaluation of the Preclinical Proton Therapy Response in Hepatocellular Carcinoma

\section{Authors list:}

Brasse David 1*, Burckel Hélène 2, Marchand Patrice 1, Rousseau Marc 1, Ouadi Ali 1, Vanstalle Marie ${ }^{1}$, Finck Christian ${ }^{1}$, Laquerriere Patrice ${ }^{1}$, Boisson Frédéric ${ }^{1}$

1 Université de Strasbourg, CNRS, IPHC UMR 7178, F-67000 Strasbourg, France

2 Institut de Cancérologie Strasbourg Europe (ICANS), UNICANCER, Paul Strauss Comprehensive Cancer Center, Radiobiology Laboratory, 67000 Strasbourg, France

*Corresponding author:

Brasse David

Institut Pluridisciplinaire Hubert Curien

23 rue du Loess 67037 Strasbourg, France

Email: david.brasse@iphc.cnrs.fr

Tel : +33388106418

Running Title: FDG and FLT Comparison for Preclinical Proton Therapy in HCC

Manuscript category: Original paper 


\begin{abstract}
Purpose

The main objective of the present study was to compare the 2-deoxy-2-[ $\left.{ }^{18} \mathrm{~F}\right]$ fluoro-D-glucose ([ $\left.\left.{ }^{18} \mathrm{~F}\right]-\mathrm{FDG}\right)$ and $3^{\prime}-\left[{ }^{18} \mathrm{~F}\right]$ fluoro-3'-deoxythymidine ( $\left.\left[{ }^{18} \mathrm{~F}\right]-\mathrm{FLT}\right)$ PET imaging biomarkers for the longitudinal follow-up of small animal proton therapy studies in the context of hepatocellular carcinoma (HCC).
\end{abstract}

\title{
Procedures
}

SK-HEP-1 cells were injected into NMRI nude mice to mimic human HCC. The behavior of $\left[{ }^{18} \mathrm{~F}\right]-\mathrm{FDG}$ and $\left[{ }^{18} \mathrm{~F}\right]-\mathrm{FLT}$ tumor uptake was evaluated after proton therapy procedures. The proton single-fraction doses were 5, 10 and $20 \mathrm{~Gy}$, with a dose rate of $10 \mathrm{~Gy} / \mathrm{min}$. The experimental protocol consisted of 8 groups of 10 mice, each group experiencing a particular dose/radiotracer condition. A reference PET exam was performed on each mouse the day before the irradiation procedure, followed by PET exams every three days up to 16 days after irradiation.

\section{Results}

$\left[{ }^{18} \mathrm{~F}\right]-\mathrm{FDG}$ uptake showed a linear dose-dependent increase in the first days after treatment $(37 \%, \mathrm{p}<0.05)$, while $\left[{ }^{18} \mathrm{~F}\right]-\mathrm{FLT}$ uptake decreased in a dose-dependent manner (e.g $21 \%$ for 5 Gy compared to $10 \mathrm{~Gy}, \mathrm{p}=1.1 \mathrm{e}-2)$. At the later time point, $\left[{ }^{18} \mathrm{~F}\right]-\mathrm{FDG}$ normalized activity showed an $85 \%$ decrease $(\mathrm{p}<0.01)$ for both 10 and 20 Gy doses and no variation for 5 Gy. Conversely, a significant $61 \%(\mathrm{p}=0.002)$ increase was observed for $\left[{ }^{18} \mathrm{~F}\right]$-FLT normalized activity at 5 Gy and no variation for higher doses.

\section{Conclusion}

We showed that the use of the $\left[{ }^{18} \mathrm{~F}\right]-\mathrm{FDG}$ and $\left[{ }^{18} \mathrm{~F}\right]-\mathrm{FLT}$ radiolabeled molecules can provide useful and complementary information for longitudinal follow-up of small animal proton therapy studies in the context of HCC. $\left[{ }^{18} \mathrm{~F}\right]-\mathrm{FDG}$ PET imaging enables a treatment monitoring 
several days / weeks postirradiation. On the other hand, $\left[{ }^{18} \mathrm{~F}\right]$-FLT could represent a good candidate to monitor the treatment few days postirradiation, in the context of hypo-fractioned and close irradiations planning. This opens new perspectives in terms of treatment efficacy verification depending on the irradiation scheme.

Keywords: PET; Proton therapy; Preclinical studies; Longitudinal study; Hepatocellular carcinoma.

\section{Introduction}

Hepatocellular carcinoma (HCC) is a type of tumor that requires a specific irradiation schedule to control its development. Radiotherapy has emerged as an alternative for patients with $\mathrm{HCC}$ when other curative modalities are not suitable [1]. In addition, various reports have described favorable clinical outcomes of proton therapy compared to traditional approaches such as Xray therapy [2].

Proton therapy centers are recruiting patients to highlight the efficacy of proton-beam therapy against advanced diseases such as $\mathrm{HCC}[3,4]$. Although proton therapy has been demonstrated to be effective in the treatment of HCC, Eggert and Greten highlighted its limited availability as the major disadvantage of this radiation modality [5], probably due to infrastructure costs and to the fact that indications are still evolving and being investigated [6].

Positron emission tomography (PET) functional imaging modality combined with computerized tomography (PET/CT) has found a well-established role before and during the radiotherapy planning process, mainly when associated with the tracer 2-deoxy-2-[ $\left.{ }^{18} \mathrm{~F}\right]$ fluoroD-glucose ([ $\left.\left.{ }^{18} \mathrm{~F}\right]-\mathrm{FDG}\right)$, such as in head-and-neck [7], hepatobiliary [8], esophageal [9] and lung malignancies [10]. PET imaging has also found a place in the evaluation of radiotherapy response [11-13] and the long-term evaluation of the effect of proton treatment [14]. 
$\left[{ }^{18} \mathrm{~F}\right]-\mathrm{FDG}$ provides a basic level of information on the tumor metabolism and can also be useful to follow the side effects of irradiation in healthy tissue due to its uptake in inflammatory tissues $[15,16] .3^{\prime}-\left[{ }^{18} \mathrm{~F}\right]$ fluoro-3'-deoxythymidine ([$\left.\left.{ }^{18} \mathrm{~F}\right]-\mathrm{FLT}\right)$, a fluorinated analog of thymidine, was developed to investigate cellular proliferation in cancer. Its uptake in cells depends on thymidine kinase (TK-1) activity and therefore on the mitotic activity of the cells, based on the thymidine salvage pathway.

Clinical trials involving $\left[{ }^{18} \mathrm{~F}\right]$-FLT PET have also demonstrated its potential as an early predictor of outcome after radiotherapy or chemotherapy [17] and have been suggested to monitor the proliferative activity of HCC [18]. It has also been proposed to assess the early response to radiation therapy due to its non-specific radiation-induced inflammation, in contrast to $\left[{ }^{18} \mathrm{~F}\right]-\mathrm{FDG}[19]$.

To effectively appreciate and promote the clinical potential benefits of proton therapy, it is necessary to understand the radiobiological properties involved. Although several biological questions have been investigated in vitro, mainly related to cell survival and proliferation, there are still ongoing studies and a need for the development of proton irradiation platforms dedicated to preclinical investigations of both in vitro experiments and in vivo small animal models [20-24].

In the present study, we used a preclinical proton therapy platform based on a $25 \mathrm{MeV}$ cyclotron. SK-HEP-1 cells mimicking human HCC were injected into mice to monitor the efficacy of the treatment using preclinical PET imaging. These cells have widely served as a model of HCC since their establishment in 1971. Despite a recent study demonstrating that SKHEP-1 should be considered an immortal human liver sinusoidal endothelial cell line instead of an HCC cell line [25], its aggressive migration [26] combined with its radioresistance made it a good candidate for the current study [27]. 
The main goal of the present study was to compare the $\left[{ }^{18} \mathrm{~F}\right]-\mathrm{FDG}$ and $\left[{ }^{18} \mathrm{~F}\right]-\mathrm{FLT}$ PET imaging radiolabeled molecules for the longitudinal follow-up of small animal proton therapy studies in HCC to highlight their potential interests to monitor the treatment depending on the irradiation scheme.

\section{Materials and Methods}

\section{Cell line and culture conditions}

The HCC cell line SK-HEP-1 was obtained from the American Type Culture Collection (HBT52, ATCC, USA). Cells were grown in Dulbecco's modified Eagle's medium (Pan Biotech, Dutscher, France) supplemented with 10\% fetal bovine serum, 4-(2-hydroxyethyl)-1piperazineethanesulfonic acid buffer, $1 \mathrm{mM}$ sodium pyruvate, $1 \mathrm{mM}$ nonessential amino acids, $100 \mathrm{U} / \mathrm{mL}$ penicillin and $100 \mu \mathrm{L} / \mathrm{ml}$ streptomycin. Cells were maintained in an incubator at $37^{\circ} \mathrm{C}$ in a humidified atmosphere.

\section{Animal model}

Six-week-old, athymic, female NMRI-Foxn1 nu/nu mice were purchased from Janvier Laboratories (Saint Berthevin, France) and housed under pathogen-free conditions.

Animal procedures were performed in accordance with experimental protocols and guidelines for the care and use of animals and approved by the Strasbourg ethics committee for animal experimentation and the French ministry in charge of research (CREMEAS, Strasbourg, France, Apafis \#261).

One week after the mice arrived, SK-HEP-1 cells $\left(10 \times 10^{6}\right)$ were injected subcutaneously into the left hindlimb of mice in $50 \mu \mathrm{L}$ of phosphate-buffered saline. The animal weight and tumor diameter were recorded three times a week during the experiment and followed up to detect any weight loss or change. The small and long axes of the tumor were measured using calipers, and the mean value was recorded as the diameter. Tumor growth occurred over a two-week period, 
during which a typical diameter of 3-10 mm was observed. Seven days post-cell injection (PCI), $90 \%$ of the tumor had reached a suitable diameter $(<6 \mathrm{~mm}$ in depth) adapted to the $25 \mathrm{MeV}$ proton beam. The mice were then randomized into several groups of 10 mice to undergo PET exams and immunohistochemical analysis.

\section{Molecule synthesis}

Pharmaceutical-grade $\left[{ }^{18} \mathrm{~F}\right]-\mathrm{FDG}$ was purchased from IBA (France). $\left[{ }^{18} \mathrm{~F}\right]$-FLT was synthesized using known radiolabeling procedures on automated radiosynthesizers with a specific activity ranging from 35 to $72 \mathrm{GBq} / \mu \mathrm{mol}$ [28] (Raytest Synchrom Evo III or Trasis AllInOne). The synthesis was controlled by high performance liquid chromatography (HPLC) (purity $>95 \%$ ) and, if necessary, gamma-thin-layer chromatography (mini-Gita, Raytest Germany). The stability over $6 \mathrm{~h}$ was assessed by HPLC, and no degradation was observed. Radiotracers were formulated in $0.9 \%$ sterile $\mathrm{NaCl}$ (veterinary grade, B. BRAUN) for i.v. injections.

\section{Irradiation setup}

The $25 \mathrm{MeV}$ proton beam was extracted from the cyclotron (TR24, ACSI, Canada) and shaped towards the tumor through a vacuum pipe. Before reaching the irradiation area, a homogeneous broad beam was obtained with an Al-scattering foil $(200 \mu \mathrm{m}$ thick $)$ set behind a $5 \mathrm{~mm} \mathrm{Al}$ collimator placed $240 \mathrm{~cm}$ upstream. The spread-out Bragg peak (SOBP) was achieved using an energy degrader consisting of $20 \mathrm{Al}$ disks of increasing thickness ranging from 147 to 2761 $\mu \mathrm{m}$, leading to linear energy transfer variation and SOBP delivery [29]. An additional $10 \mathrm{~mm}$ collimator was located after the degrader to finalize the beam shaping (Fig. 1). The dose delivered to the tumor was set up both by the beam intensity and the irradiation duration, controlled with a beam kicker located between the source and the entrance of the cyclotron cavity, allowing fast beam shutoff. More information on the preclinical proton beamline and the associated dosimetry can be found in [30]. During the whole irradiation procedure, each 
mouse was anaesthetized using IP injection of ketamine $(65 \mathrm{mg} / \mathrm{kg}) / x y l a z i n e ~(10 \mathrm{mg} / \mathrm{kg})$. The mouse body temperature was maintained at $36 \pm 1{ }^{\circ} \mathrm{C}$ using a vivarium heating lamp.

\section{Description of the experimental protocol}

For the evaluation of both molecules, the experimental protocol consisted of eight groups of ten mice. Each group represented a particular dose/radiotracer condition. The proton singlefraction doses were 5, 10 and $20 \mathrm{~Gy}$, with a dose rate of $10 \mathrm{~Gy} / \mathrm{min}$, plus a control group with no dose delivered for each molecule. The delivered doses were chosen to range from low to high dose and according to previous preclinical results obtained with external beam irradiation $[19,31]$. For each irradiated mouse, the dose was uniformly distributed in a cylindrical volume of $10 \mathrm{~mm}$ diameter and $5 \mathrm{~mm}$ depth, centered in the tumor. A reference time point was defined as the day before the irradiation procedure, seven days PCI, at which a reference PET exam was acquired for each mouse. Additional PET exams were then performed on each mouse every three days up to 16 days after irradiation.

\section{Small animal PET imaging protocol}

Animals were scanned with a dedicated preclinical PET (IRIS PET, Inviscan). The injected activities for both radiolabeled molecules were $10 \mathrm{MBq}$ via the tail vein. Static image acquisitions were performed 45 minutes ([ $\left.{ }^{18} \mathrm{~F}\right]-\mathrm{FDG}$ groups) and 90 minutes ([ $\left.{ }^{18} \mathrm{~F}\right]$-FLT groups) after injection. The time between the radiotracer injection and the different PET exams was investigated and was chosen to optimize the tumor-to-background uptake ratio and according to the literature. To optimize $\left[{ }^{18} \mathrm{~F}\right]$-FDG tumor uptake, the mice were fasted overnight and kept warm and anesthetized during the 45-minute biodistribution [32]. During the entire exam, each mouse was maintained under isoflurane anesthesia (2\%, MINERVE company).

Two photons detected within a 5 ns coincidence timing window and with an energy ranging from 250 to $750 \mathrm{keV}$ were defined as a coincidence. For each PET exam, the coincidences were acquired for 10 minutes. Data were reconstructed into a $201 \times 201 \times 120$ volume using the 
iterative 3D ordered-subset expectation-maximization algorithm. The resulting voxel size was equal to $0.42 \mathrm{~mm}$ in the transverse plane while the slice thickness was equal to $0.855 \mathrm{~mm}$. PET data were fully corrected for normalization, random coincidences, radioactive decay and dead time during the reconstruction process. No attenuation and scatter corrections were applied.

\section{PET Data analysis}

PET data analyses were performed using the AMIDE software package [33]. A focal nonphysiologic increase in radiotracer uptake in the area of tumor development was considered positive PET. An ellipsoid was manually drawn on the tumor, large enough to account for the entire radiotracer uptake of the tumor. The standardized uptake value (SUV) was calculated for all voxels and the resulting highest value was defined as the SUVmax. Inside the volume, a region of interest (ROI) was defined by all the voxels with a value greater than $40 \%$ of the SUVmax value. The SUV mean value (SUVmean) inside the ROI was calculated, as was the functional volume, defined as the sum of all the voxels in the ROI. Activity was also calculated as the product between the mean voxel value calculated inside the ROI $(\mathrm{Bq} / \mathrm{mL})$ and the functional volume $(\mathrm{mL})$. The functional volume and the functional activity are equivalent to the metabolic tumor volume and the total lesion glycolysis specifically defined for $\left[{ }^{18} \mathrm{~F}\right]-\mathrm{FDG}$. To investigate the behavior of the different calculated figures of merit after irradiation, all the calculated values were divided by the values obtained at the reference time point, seven days PCI.

\section{Immunohistochemical procedure}

Three groups of 40 mice each were involved in the immunohistochemical procedure. Each group represented a time point and consisted in 10 mice per irradiation condition (control, 5 Gy, 10 Gy and 20 Gy). Mice were sacrificed at accurate times before and after irradiation (-1, +3 and +16 days). Tumor samples were removed and fixed in buffered formalin for at least $24 \mathrm{~h}$, dehydrated and paraffin embedded. Standard $5 \mu \mathrm{m}$ sections were sliced, and histological 
staining was carried out with hematoxylin-eosin to analyze the mode of organization of the cells, the size and shape of the tumor and the degree of tissue damage. The expression of the Ki-67 antigen was detected by immunohistochemistry for use as a marker of tumor proliferation. This antigen was detected using the mouse monoclonal antibody against Ki-67 (clone MIB1, DAKO M7240, 1/50 e dilution). The quantitative evaluation was performed by counting with ImageJ [34] after observing tissue sections at high magnification (x40) and determining the number of positive tumor cells relative to the total number of tumor cells present on the same field of microscopic observation. The results were evaluated by counting the number of nuclei positive for immunostaining out of a total of 1000 tumor cells (10 to 20 observation fields).

\section{Statistical analysis}

We compared each variable between the groups using the Mann-Whitney rank test function. Statistical calculation was performed using the Python programming platform associated with the Pandas and SciPy libraries $[35,36]$. The level of significance was $p<0.05$. Descriptive statistics and box plots were mainly used for data analysis.

\section{Results}

Visual analysis of reconstructed images demonstrated a better tumor-to-surrounding background uptake ratio for $\left[{ }^{18} \mathrm{~F}\right]$-FLT compared to $\left[{ }^{18} \mathrm{~F}\right]$-FDG leading to a better delineation for VOI placement (Fig. 2). The SUVmean, the functional volume and the resulting activity were calculated and normalized to evaluate the $\left[{ }^{18} \mathrm{~F}\right]-\mathrm{FDG}$ and the $\left[{ }^{18} \mathrm{~F}\right]$-FLT uptakes under control and irradiation conditions.

\section{In vivo characterization of $\left[{ }^{18} \mathrm{~F}\right]-F D G$ uptake}

The results obtained for the control condition are shown in in Figures 3a-c. 
Between 7 and 11 days PCI, a first phase could be defined as a tumor expansion period, when a decrease in the normalized SUVmean $(p=3 e-4)$ and an increase in the normalized functional volume $(p=3 e-4)$ were observed, resulting in stable normalized activity values $(p=3 e-1)$. In the second phase, a decrease in the normalized functional volume up to 17 days PCI ( $p=4.8 \mathrm{e}-2)$ was noted. The increase in the normalized SUVmean in the same period was also statistically significant ( $\mathrm{p}=1 \mathrm{e}-2)$. For the last phase, starting between 14 and 17 days PCI, a decrease in the normalized SUVmean $(p=2 e-2)$ and an increase in the normalized functional volume were highlighted, indicating tumor recovery $(\mathrm{p}=1 \mathrm{e}-3)$.

The results for $\left[{ }^{18} \mathrm{~F}\right]$-FDG PET imaging after proton irradiation are given in Figure 3d-f, where only the normalized activity is presented. While a decrease in the normalized activity after 10 $(\mathrm{p}=2 \mathrm{e}-4)$ and 20 Gy irradiation $(\mathrm{p}=1 \mathrm{e}-2)$ over the examination period was observed, the normalized activity decreased at the beginning of the experimental protocol ( $p=3 e-2$ between 3 and 6 days after irradiation) and remained stable after 9 days postirradiation in the 5 Gy group. The difference between 5 Gy and the other irradiation doses was amplified after 9 days. No significant differences were observed between the control group and the tumors irradiated with 5 Gy.

\section{In vivo characterization of $\left[{ }^{18}\right.$ F]-FLT uptake}

The results obtained for the control condition are shown in Figure 4a-c.

Between the $7^{\text {th }}$ and the $14^{\text {th }}$ day PCI, a growth phase was characterized by an increase in both the normalized SUVmean $(p=4 e-2)$ and the normalized functional volume ( $p=4 e-3)$, resulting in an increase in the normalized activity $(2.63 \pm 1.5, \mathrm{p}=2 \mathrm{e}-4)$. Despite a visual increase of the SUVmean between the 11th and the 14th day PCI, this obtained difference was not statistically significant $(\mathrm{p}=6 \mathrm{e}-2)$. The behavior of the 3 figures of merit was stable after day 14 .

Figures $4 \mathrm{~d}-\mathrm{f}$ present the normalized activities of $\left[{ }^{18} \mathrm{~F}\right]$-FLT tumor uptake after 5, 10 and $20 \mathrm{~Gy}$ proton irradiation. Three days after irradiation, an increase in normalized $\left[{ }^{18} \mathrm{~F}\right]$-FLT activity in 
the 5 Gy group was observed ( $p=1 \mathrm{e}-4)$, while no change was seen in the 10 Gy group $(p=4 e-$ 1 ), and a decrease was seen in the 20 Gy group $(p=2 e-2)$.

After these 3 days, the normalized $\left[{ }^{18} \mathrm{~F}\right]-\mathrm{FLT}$ activities remained constant, with no significant difference in any proton-dose group except a significant decrease after 20 Gy the last day of the experiment ( $p=4.7 \mathrm{e}-2$ between 6 and 16 days after irradiation).

A difference between the normalized $\left[{ }^{18} \mathrm{~F}\right]$-FLT activity in the 5 and 20 Gy groups three days after irradiation was observed $(p=2 e-3)$. A significant difference was observed between the mice irradiated with 10 or 20 Gy and the control mice from six days after irradiation ( $p=2 e-3$ ) until the end of the experimental protocol $(p=3 e-3)$.

Conversely, no significant difference was observed between the data obtained in the 5 Gy group and the control group except at 9 days after irradiation, where the calculated median activity in the 5 Gy group (1.9) was significantly lower than the median in the control mice (2.5) ( $p=4.6 \mathrm{e}-$ 2).

\section{Comparison of both molecules at an early stage after irradiation}

The interpretation of $\left[{ }^{18} \mathrm{~F}\right]-\mathrm{FDG}$ uptake in the early days following irradiation was difficult because $\left[{ }^{18} \mathrm{~F}\right]$-FDG responds to inflammatory changes. Three days after irradiation, the normalized SUVmax calculated after $\left[{ }^{18} \mathrm{~F}\right]-\mathrm{FDG}$ injection increased by a factor proportional to the irradiation dose (Figure 5a). The p-values were 4.8e-2 for the control group compared to the 5 Gy group, 3.6e-2 for 5 Gy compared to 10 Gy and $2.8 \mathrm{e}-2$ for 10 Gy compared to 20 Gy. We observed a decrease of the normalized functional volume when the irradiation dose increased leading to a stable normalized activity (data not shown).

The observed behavior of $\left[{ }^{18} \mathrm{~F}\right]-\mathrm{FDG}$ uptake at the early stage was counterbalanced by the decrease in $\left[{ }^{18} \mathrm{~F}\right]$-FLT uptake as the proton dose increased (Figure $5 \mathrm{~b}$ ). Going from 5 to $10 \mathrm{~Gy}$, the median of the normalized SUVmax fell by $21 \%(\mathrm{p}=1.1 \mathrm{e}-2)$, and the drop was $36 \%$ when 5 Gy was compared to $20 \mathrm{~Gy}(\mathrm{p}=1.2 \mathrm{e}-3)$. We also observed a decrease of the normalized 
functional volume leading to a decrease of the normalized activity when the irradiation dose increase (data not shown).

\section{Evaluation of the Ki-67 index}

Variations in the expression of the Ki-67 index as a proliferation marker are presented in Figure 6. Nonirradiated SK-HEP-1 tumors showed an important Ki-67 proliferation index between 65 and $75 \%$, regardless of the day of collection. Three days after irradiation, irradiated tumors presented a significant decrease in their Ki-67 indexes of approximately $40 \%$, irrespective of the irradiation dose $(p=1 e-4)$. Then, between 3 and 16 days postirradiation, the Ki-67 index of the tumors irradiated with 5 Gy remained stable, while a decrease in Ki-67 was observed in the 10 and 20 Gy groups. Sixteen days postirradiation, Ki-67 varied from $42 \%$ after 5 Gy to $26 \%$ after 10 Gy to $10 \%$ after 20 Gy irradiation. At the end of the experimental protocol, a significant dose-dependent decrease was observed in the Ki-67 of tumor cells between the 5 and 20 Gy groups $(p=1 e-4)$.

\section{Discussion}

In this work, we used static PET imaging to evaluate the behavior of metabolism and cell proliferation in tumors after preclinical proton therapy procedures. The main objective was to compare the $\left[{ }^{18} \mathrm{~F}\right]-\mathrm{FDG}$ and $\left[{ }^{18} \mathrm{~F}\right]-\mathrm{FLT}$ radiolabeled molecules to monitor the treatment in an HCC context.

The results obtained for each dose/radiotracer condition showed important variations per analyzed day. This variation is due not only to statistical fluctuations ( $\mathrm{N}=10$ per condition) but also to factors related to the imaging technique itself, the quantification approach and other animal-specific parameters. In our study, we tried to reduce these factors by optimizing the experimental conditions [37] and by normalizing each investigated figure of merit to a reference value measured before the irradiation. To overcome some limitations of PET SUV 
measurements, we also measured the functional volume to derive an activity value representative of the overall metabolism and the cell proliferation behavior at the tumor level. The single-fraction procedure used in our study is not fully representative of the irradiation protocol used for human therapy, where radiation is usually administered to patients in multiple fractions. However, clinical studies on liver demonstrated high rates of local tumor control using high-dose hypofractionated stereotactic body radiation therapy [38]. Therefore, our protocol mimics the effect of a high dose delivered on a mouse model of HCC. Additional experiments have to be addressed before extending our conclusions to patients.

$\left[{ }^{18} \mathrm{~F}\right]$-FDG uptake showed a linear dose-dependent increase in the first days after treatment $(37 \%, \mathrm{p}<0.05)$, while $\left[{ }^{18} \mathrm{~F}\right]-\mathrm{FLT}$ uptake decreased in a dose-dependent manner (e.g $21 \%$ for 5 Gy compared to $10 \mathrm{~Gy}, \mathrm{p}=1.1 \mathrm{e}-2)$. We made the assumption that the increase of $\left[{ }^{18} \mathrm{~F}\right]-\mathrm{FDG}$ uptake in the first days following the irradiation was due to potential inflammatory changes. However, previous study showed that in vitro radiation of tumor cells using X-rays could result in an increase of $\left[{ }^{18} \mathrm{~F}\right]$-FDG uptake without presenting inflammatory cells [39]. Further investigation on proton associated with SK-HEP-1 cells should be conducted to clarify this specific point.

At the later time point, $\left[{ }^{18} \mathrm{~F}\right]-\mathrm{FDG}$ normalized activity showed an $85 \%$ decrease $(\mathrm{p}<0.01)$ for both 10 and 20 Gy doses and no variation for 5 Gy. Conversely, a significant $61 \%(p=0.002)$ increase was observed for $\left[{ }^{18} \mathrm{~F}\right]$-FLT normalized activity at 5 Gy and no variation for higher doses. The current results obtained with $\left[{ }^{18} \mathrm{~F}\right]-\mathrm{FLT}$ are consistent with those already published by Lin et al, demonstrating the value of using $\left[{ }^{18} \mathrm{~F}\right]$-FLT to monitor the early response of cell proliferation to proton therapy in a colon carcinoma cell line [19]. In the current study, we observed the same behavior of $\left[{ }^{18} \mathrm{~F}\right]-\mathrm{FLT}$ activity and proliferation activity (Ki-67) two weeks after irradiation. Previous work also demonstrated a significant correlation of $\left[{ }^{18} \mathrm{~F}\right]$-FLT with the proliferating fraction of $\mathrm{HCC}$ as assessed by Ki-67 immunohistochemistry [18, 40]. 
Considering that tumor cell proliferation is a mechanism of therapy resistance, the use of $\left[{ }^{18} \mathrm{~F}\right]-$ FLT can provide an effective decision tool for treatment management.

The literature on the use of $\left[{ }^{18} \mathrm{~F}\right]$-FLT in the clinical routine reveals high background signals in the liver, which can be a potential drawback of using this molecule in HCC [41]. Despite this physiologic retention in the liver, Eckel et al [18] reported a median 20\% higher focal uptake by malignant tumors than the surrounding liver, leading to a $69 \%$ sensitivity for the detection of HCC. To optimize the tumor-to-liver contrast, Sharma et al proposed in a pilot study to classified each voxel according to their time activity curve [42]. However, they showed that the high and variable SUV limited the improvement of tumor visualization. During the last decade, several approaches have been proposed to improve the sensitivity of PET/CT in HCC diagnosis $[43,44]$. However, according to the European Association for the Study of the liver, there is no single radiolabeled molecule recommended for either diagnosis or response to therapy procedures in HCC [45]. The development of new probes and new procedures should not only improve the diagnosis but also the therapy response assessment.

\section{Conclusions}

We showed that the use of the $\left[{ }^{18} \mathrm{~F}\right]-\mathrm{FDG}$ and $\left[{ }^{18} \mathrm{~F}\right]$-FLT radiolabeled molecules can provide useful and complementary information for longitudinal follow-up of small animal proton therapy studies in the context of HCC. Our conclusions were drawn from the results obtained from HCC cell line SK-HEP-1. $\left[{ }^{18} \mathrm{~F}\right]-\mathrm{FDG}$, a reference molecule for PET imaging, enables a treatment monitoring several days / weeks postirradiation. On the other hand, $\left[{ }^{18} \mathrm{~F}\right]-\mathrm{FLT}$ could represent a good candidate to monitor the treatment few days postirradiation, in the context of hypo-fractioned and close irradiations planning. This opens new perspectives in terms of treatment efficacy verification depending on the irradiation scheme. 


\section{Acknowledgments}

This work was partly supported by ITMO Cancer AVIESAN (Alliance Nationale pour les Sciences de la Vie et de la Santé, National Alliance for Life Sciences and Health) within the framework of the Cancer Plan and by $\mathrm{IN}_{2} \mathrm{P}_{3}$. The authors wish to thank Bruno Jessel, Lionel Thomas, Jacky Schuler, Michel Pellicioli, Julie Constanzo and Cédric Mathieu for their help in performing all the experiments. We also thank all the members of the mechanical workshop involved in the development of the beam line.

\section{Author contributions}

All authors discussed and conceived the generic concept and reviewed the manuscript. D.B. wrote the main part of the manuscript. P.M. and A.O. contributed to the PET experiments. M.R., M.V. and C.F. participated in the setup of the preclinical proton beamline and the associated dosimetry. H.B. performed the cell culture and the immunohistochemical procedure and analyses. P.L. performed the statistical analyses. F.B and D.B analyzed the PET data.

\section{Conflict of Interest}

The authors declare that they have no conflict of interest.

\section{References}

1. Ohri N, Dawson LA, Krishnan S, et al (2016) Radiotherapy for Hepatocellular Carcinoma: New Indications and Directions for Future Study. J Natl Cancer Inst 108

2. Yoo GS, Yu JI, Park HC (2018) Proton therapy for hepatocellular carcinoma: Current knowledges and future perspectives. World J Gastroenterol 24:3090-3100.

3. Klein J, Dawson LA (2013) Hepatocellular carcinoma radiation therapy: review of evidence and future opportunities. Int J Radiat Oncol Biol Phys 87:22-32.

4. Dionisi F, Widesott L, Lorentini S, Amichetti M (2014) Is there a role for proton therapy 
in the treatment of hepatocellular carcinoma? A systematic review. Radiother Oncol 111:1-10. 5. Eggert T, Greten TF (2017) Current Standard and Future Perspectives in Non-Surgical Therapy for Hepatocellular Carcinoma. Digestion 96:1-4.

6. Yeung RH, Chapman TR, Bowen SR, Apisarnthanarax S (2017) Proton beam therapy for hepatocellular carcinoma. Expert Rev Anticancer Ther 17:911-924.

7. Grégoire V, Thorwarth D, Lee JA (2018) Molecular Imaging-Guided Radiotherapy for the Treatment of Head-and-Neck Squamous Cell Carcinoma: Does it Fulfill the Promises? Semin Radiat Oncol 28:35-45.

8. Bajpai S, Kambadakone A, Guimaraes AR, et al (2015) Image-guided Treatment in the Hepatobiliary System: Role of Imaging in Treatment Planning and Posttreatment Evaluation. Radiographics 35:1393-1418.

9. Huang Y-C, Lu H-I, Huang S-C, et al (2017) FDG PET using SUVmax for preoperative T-staging of esophageal squamous cell carcinoma with and without neoadjuvant chemoradiotherapy. BMC Med Imaging 17:1.

10. Cremonesi M, Gilardi L, Ferrari ME, et al (2017) Role of interim 18F-FDG-PET/CT for the early prediction of clinical outcomes of Non-Small Cell Lung Cancer (NSCLC) during radiotherapy or chemo-radiotherapy. A systematic review. Eur J Nucl Med Mol Imaging $44: 1915-1927$.

11. Rafat M, Ali R, Graves EE (2015) Imaging radiation response in tumor and normal tissue. Am J Nucl Med Mol Imaging 5:317-332

12. Cliffe H, Patel C, Prestwich R, Scarsbrook A (2017) Radiotherapy response evaluation using FDG PET-CT-established and emerging applications. Br J Radiol 90:20160764.

13. Decazes P, Thureau S, Dubray B, Vera P (2018) How to use PET/CT in the evaluation of response to radiotherapy. Q J Nucl Med Mol Imaging 62:152-164.

14. Ryttlefors M, Danfors T, Latini F, et al (2016) Long-term evaluation of the effect of 
hypofractionated high-energy proton treatment of benign meningiomas by means of (11)C-Lmethionine positron emission tomography. Eur J Nucl Med Mol Imaging 43:1432-1443.

15. van Elmpt W, Ollers M, Dingemans A-MC, et al (2012) Response assessment using 18F-FDG PET early in the course of radiotherapy correlates with survival in advanced-stage non-small cell lung cancer. J Nucl Med 53:1514-1520.

16. Pommier P, Touboul E, Chabaud S, et al (2010) Impact of (18)F-FDG PET on treatment strategy and 3D radiotherapy planning in non-small cell lung cancer: A prospective multicenter study. AJR Am J Roentgenol 195:350-355.

17. Hoeben BAW, Troost EGC, Span PN, et al (2013) 18F-FLT PET during radiotherapy or chemoradiotherapy in head and neck squamous cell carcinoma is an early predictor of outcome. J Nucl Med 54:532-540.

18. Eckel F, Herrmann K, Schmidt S, et al (2009) Imaging of proliferation in hepatocellular carcinoma with the in vivo marker 18F-fluorothymidine. J Nucl Med 50:1441-1447.

19. Lin C, Kume K, Mori T, et al (2015) Predictive Value of Early-Stage Uptake of 3'Deoxy-3'-18F-Fluorothymidine in Cancer Cells Treated with Charged Particle Irradiation. J Nucl Med 56:945-950.

20. Ford E, Emery R, Huff D, et al (2017) An image-guided precision proton radiation platform for preclinical in vivo research. Phys Med Biol 62:43-58.

21. Almeida IP, Vaniqui A, Schyns LE, et al (2019) Exploring the feasibility of a clinical proton beam with an adaptive aperture for pre-clinical research. Br J Radiol 92:20180446.

22. Greubel C, Assmann W, Burgdorf C, et al (2011) Scanning irradiation device for mice in vivo with pulsed and continuous proton beams. Radiat Environ Biophys 50:339-344.

23. Meyer J, Stewart RD, Smith D, et al (2017) Biological and dosimetric characterisation of spatially fractionated proton minibeams. Phys Med Biol 62:9260-9281.

24. Vanstalle M, Constanzo J, Finck C (2019) Investigation of Optimal Physical Parameters 
for Precise Proton Irradiation of Orthotopic Tumors in Small Animals. Int J Radiat Oncol Biol Phys 103:1241-1250.

25. Tai Y, Gao J-H, Zhao C, et al (2018) SK-Hep1: not hepatocellular carcinoma cells but a cell model for liver sinusoidal endothelial cells. Int J Clin Exp Pathol 11:2931-2938

26. Eun JR, Jung YJ, Zhang Y, et al (2014) Hepatoma SK Hep-1 cells exhibit characteristics of oncogenic mesenchymal stem cells with highly metastatic capacity. PLoS One 9:e110744.

27. Altmeyer A, Jung AC, Ignat M, et al (2010) Pharmacological enhancement of autophagy induced in a hepatocellular carcinoma cell line by high-LET radiation. Anticancer Res 30:303310

28. Marchand P, Ouadi A, Pellicioli M, et al (2016) Automated and efficient radiosynthesis of [(18)F]FLT using a low amount of precursor. Nucl Med Biol 43:520-527.

29. Vanstalle M, Constanzo J, Karakaya Y, et al (2018) Analytical dose modeling for preclinical proton irradiation of millimetric targets. Med Phys 45:470-478.

30. Constanzo J, Vanstalle M, Finck C, et al (2019) Dosimetry and characterization of a 25$\mathrm{MeV}$ proton beam line for preclinical radiobiology research. Med Phys 46:2356-2362.

31. Wilson GD, Thibodeau BJ, Fortier LE, et al (2014) Glucose metabolism gene expression patterns and tumor uptake of ${ }^{18} \mathrm{~F}$-fluorodeoxyglucose after radiation treatment. Int $\mathrm{J}$ Radiat Oncol Biol Phys 90:620-627.

32. Fueger BJ, Czernin J, Hildebrandt I, et al (2006) Impact of animal handling on the results of 18F-FDG PET studies in mice. J Nucl Med 47:999-1006

33. Loening AM, Gambhir SS (2003) AMIDE: a free software tool for multimodality medical image analysis. Mol Imaging 2:131-137.

34. Schneider CA, Rasband WS, Eliceiri KW (2012) NIH Image to ImageJ: 25 years of image analysis. Nat Methods 9:671-675.

35. McKinney W (2010) Data Structures for Statistical Computing in Python. Proceedings 
of the 9th Python in Science Conference 56-61.

36. Virtanen P, Gommers R, Oliphant TE, et al (2020) SciPy 1.0: fundamental algorithms for scientific computing in Python. Nat Methods 17:261-272.

37. Kuntner C, Stout DB (2014) Quantitative preclinical PET imaging: opportunities and challenges. Front Phys 2:1-12

38. Schaub SK, Hartvigson PE, Lock MI, et al (2018) Stereotactic Body Radiation Therapy for Hepatocellular Carcinoma: Current Trends and Controversies. Technol Cancer Res Treat 17.

39. Higashi K, Clavo AC, Wahl RL (1993) In vitro assessment of 2-fluoro-2-deoxy-Dglucose, L-methionine and thymidine as agents to monitor the early response of a human adenocarcinoma cell line to radiotherapy. J Nucl Med 34:773-779

40. Chalkidou A, Landau DB, Odell EW, et al (2012) Correlation between Ki-67 immunohistochemistry and 18F-fluorothymidine uptake in patients with cancer: A systematic review and meta-analysis. Eur J Cancer 48:3499-3513.

41. Tehrani OS, Shields AF (2013) PET imaging of proliferation with pyrimidines. J Nucl Med 54:903-912.

42. Sharma R, Inglese M, Dubash S, et al (2020) Monitoring Response to Transarterial Chemoembolization in Hepatocellular Carcinoma Using 18F-Fluorothymidine PET. Journal of Nuclear Medicine 61:1743-1748.

43. Ronot M, Clift AK, Vilgrain V, Frilling A (2016) Functional imaging in liver tumours. Journal of Hepatology 65:1017-1030.

44. Lu R-C, She B, Gao W-T, et al (2019) Positron-emission tomography for hepatocellular carcinoma: Current status and future prospects. World J Gastroenterol 25:4682-4695.

45. European Association for the Study of the Liver. Electronic address: easloffice@easloffice.eu, European Association for the Study of the Liver (2018) EASL 
Clinical Practice Guidelines: Management of hepatocellular carcinoma. J Hepatol 69:182-236. 


\section{Figure Legends}

Fig. 1 Picture of the horizontal beam line, including the rotating wheel used to degrade the proton energy and the collimator plus the scattering foil to shape the beam.

Fig. 2 Coronal sections of mice scan after (a) [18F]-FDG injection and (b) [18F]-FLT injection to show changes in SUV for tumor uptake during the experimental protocol. The arrow indicates the location of the tumor.

Fig. 3 Results of $\left[{ }^{18} \mathrm{~F}\right]$-FDG uptake. Mice $(\mathrm{N}=10)$ were imaged 7 days PCI, treated on day 8 , and then imaged again 3, 6, 9, 13, and 16 days postirradiation. The first row illustrates the results obtained for the control group in terms of (a) the normalized SUVmean, (b) the normalized functional volume, (c) the normalized activity. The second row represents the calculated normalized activities for the (d) $5 \mathrm{~Gy}$, (e) $10 \mathrm{~Gy}$, (f) $20 \mathrm{~Gy}$ groups. The dashed line represents the normalized activity calculated for the control group (imported from $(\mathbf{c})$ ). The * marker indicates a significant difference $(p<0.05)$. The mean and median values are represented by triangles and horizontal lines, respectively.

Fig. 4 Results of $\left[{ }^{18} \mathrm{~F}\right]$-FLT uptake. Mice $(\mathrm{N}=10)$ were imaged 7 days PCI, treated on day 8 , and then imaged again 3,6,9,13, and 16 days postirradiation. The first row illustrates the results obtained for the control group in terms of (a) the normalized SUVmean, (b) the normalized functional volume, (c) the normalized activity. The second row represents the calculated normalized activities for the (d) $5 \mathrm{~Gy}$, (e) $10 \mathrm{~Gy}$, (f) 20 Gy groups. The dashed line represents the normalized activity calculated for the control group (imported from $(\mathbf{c}))$. The * 
marker indicates a significant difference $(\mathrm{p}<0.05)$. The mean and median values are represented by triangles and horizontal lines, respectively.

Fig. 5 (a) Normalized SUV max of $\left[{ }^{18} \mathrm{~F}\right]$-FDG uptake and (b) $\left[{ }^{18} \mathrm{~F}\right]$-FLT uptake in the different proton-dose groups 3 days after irradiation. The * marker indicates a significant difference $(\mathrm{p}$ $<0.05)$. The mean and median values are represented by triangles and horizontal lines, respectively.

Fig. 6 Immunohistochemical evaluation of the Ki-67 cell proliferation index in SK-HEP-1 xenografts after different irradiation doses (NI: nonirradiated; 5, 10 and $20 \mathrm{~Gy}$ ) and on different days $(-1,3$ and 16$)$ before and after irradiation. Mean percentage $( \pm S D)$ value of Ki-67-positive SK-HEP-1 cells among 1,000 cancer cells scored in 10 tumors per condition. The $*$ marker indicates a significant difference $(\mathrm{p}<0.05)$. 


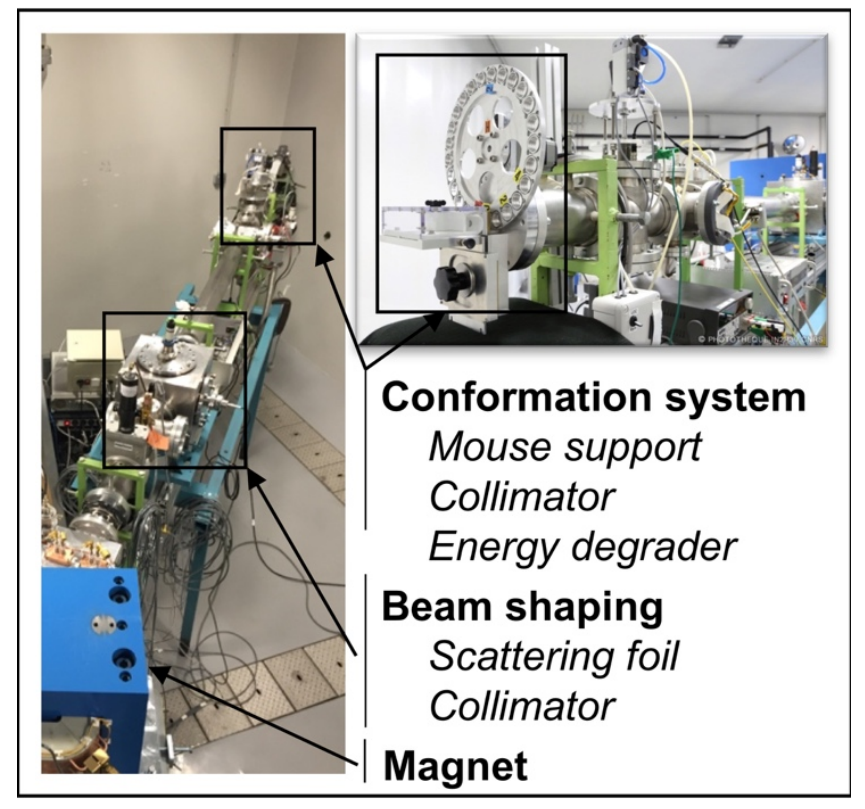

Figure 1
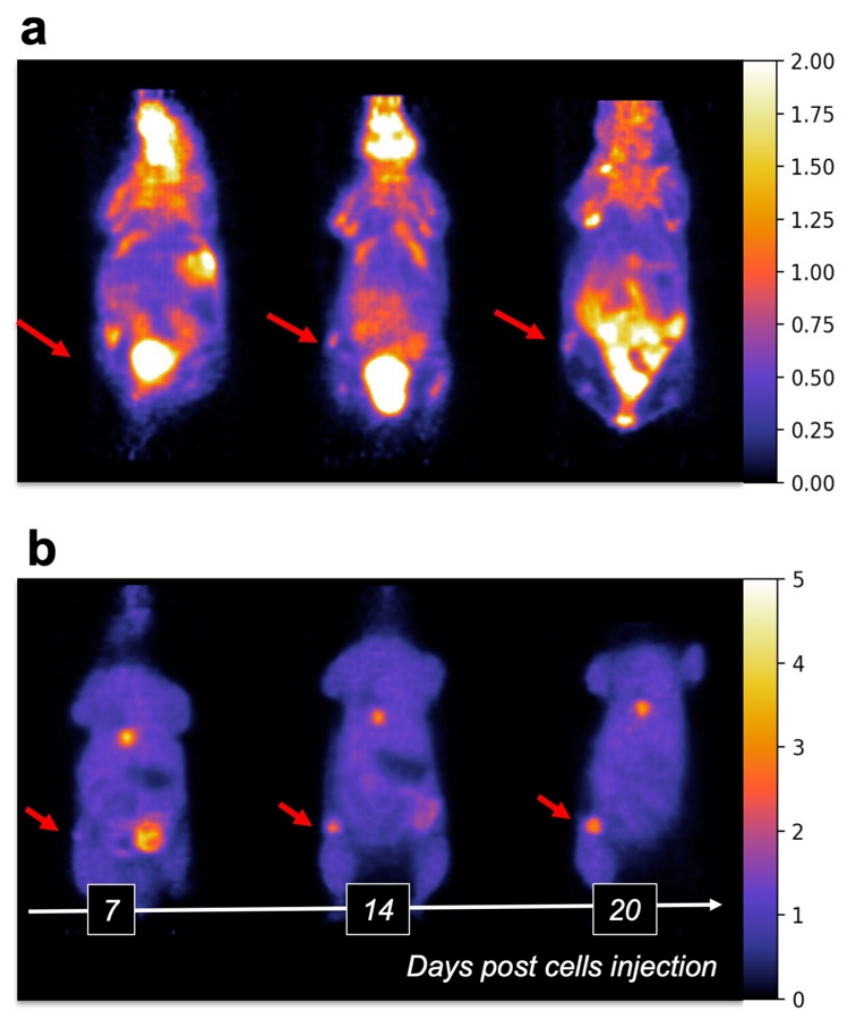

Figure 2 


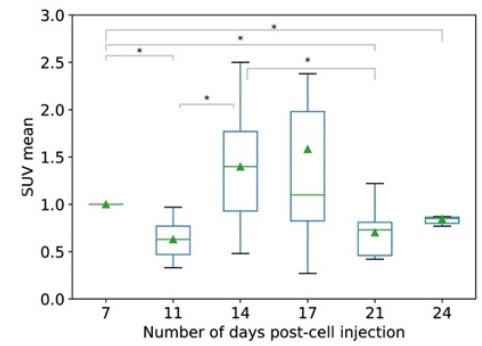

d

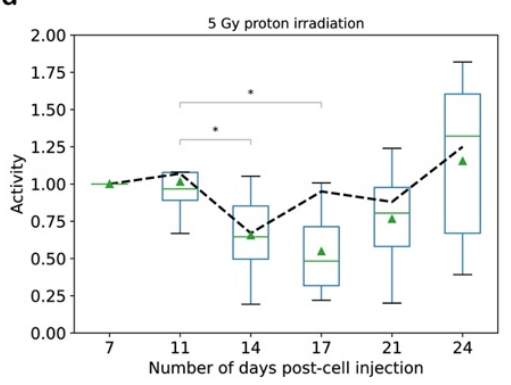

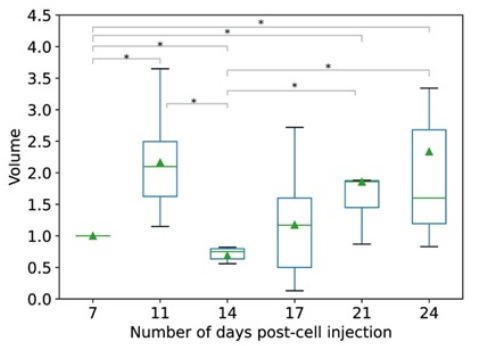

e

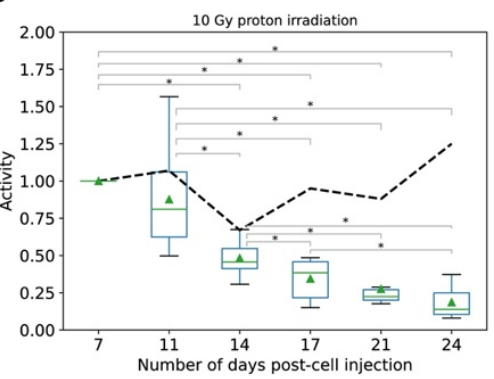

C

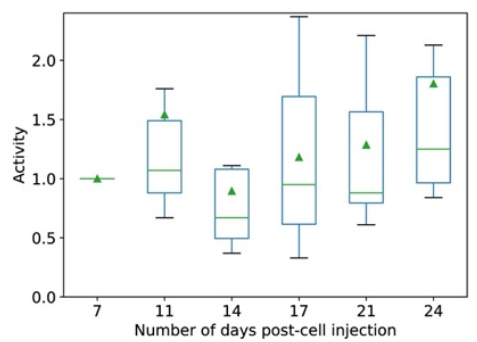

f

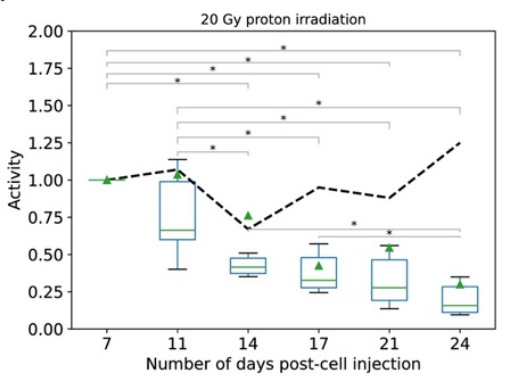

Figure 3

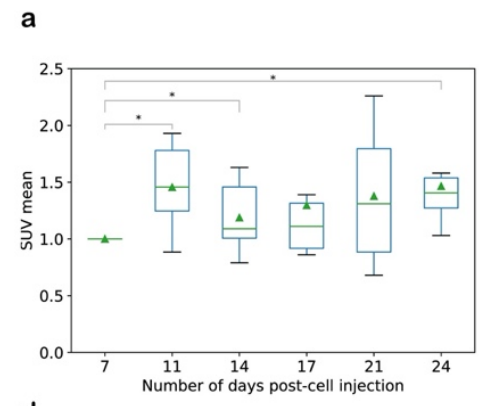

d

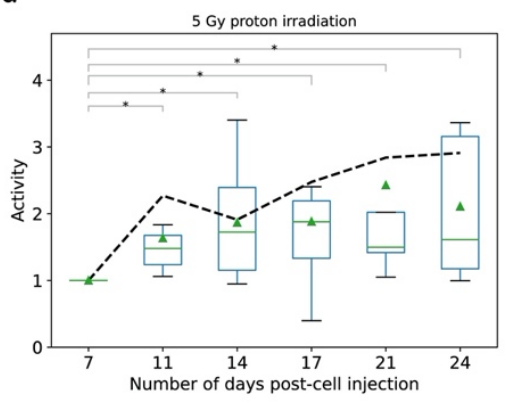

b

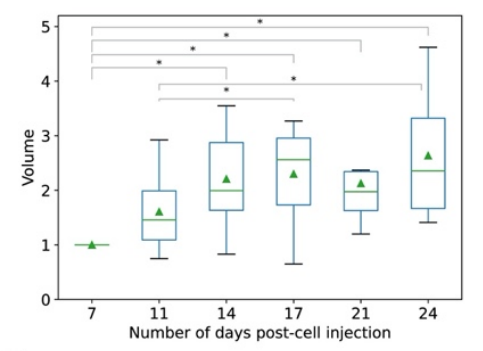

e

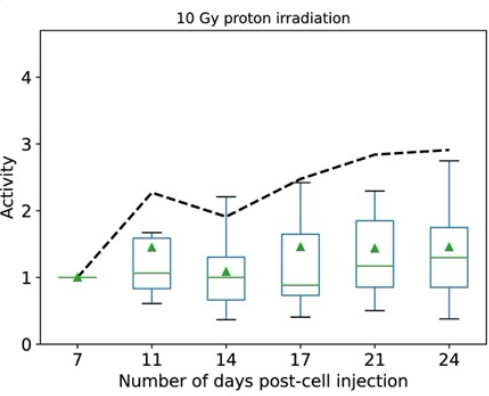

c

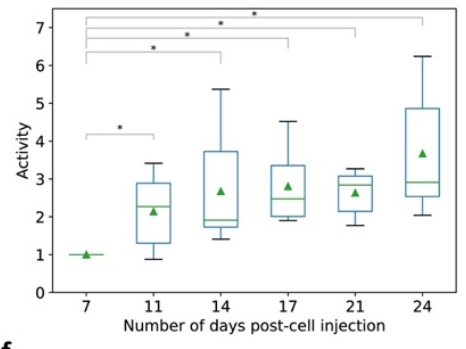

20 Gy proton irradiation

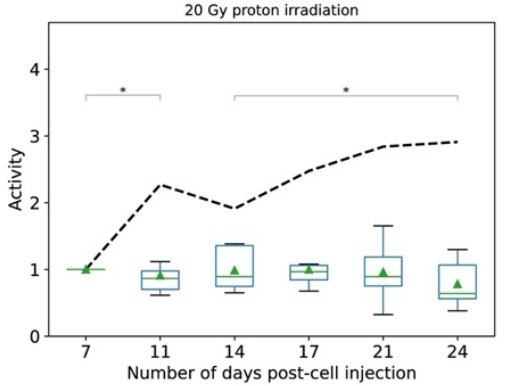

Figure 4 

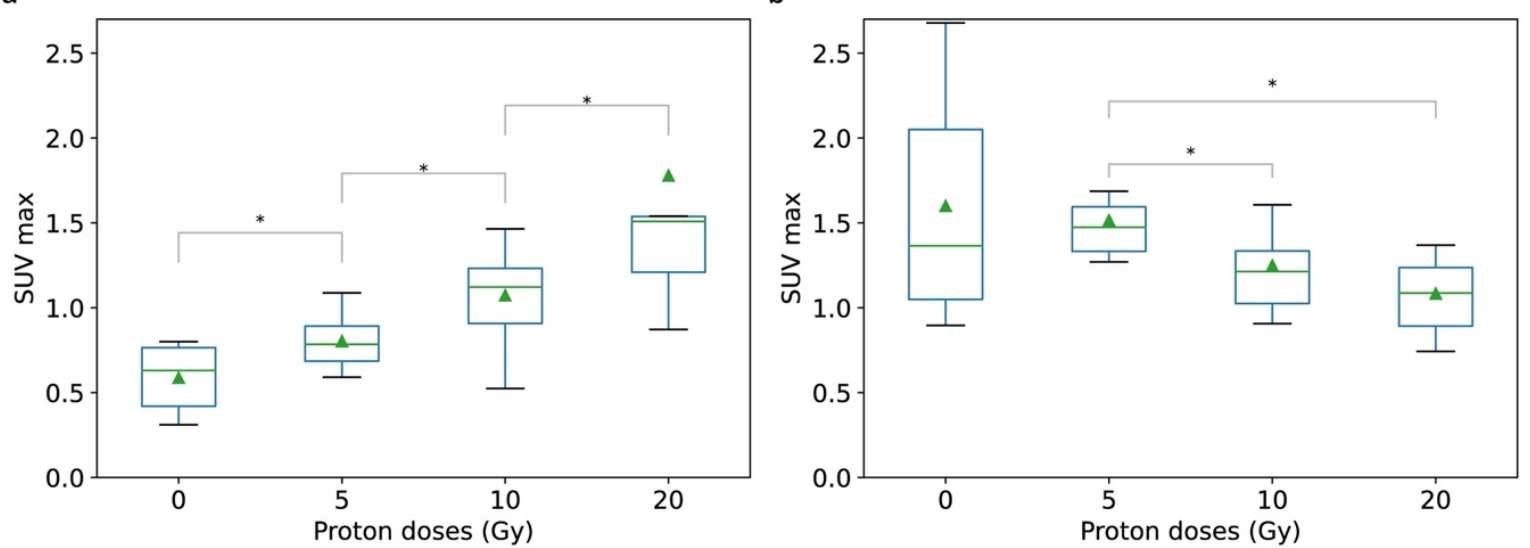

Figure 5

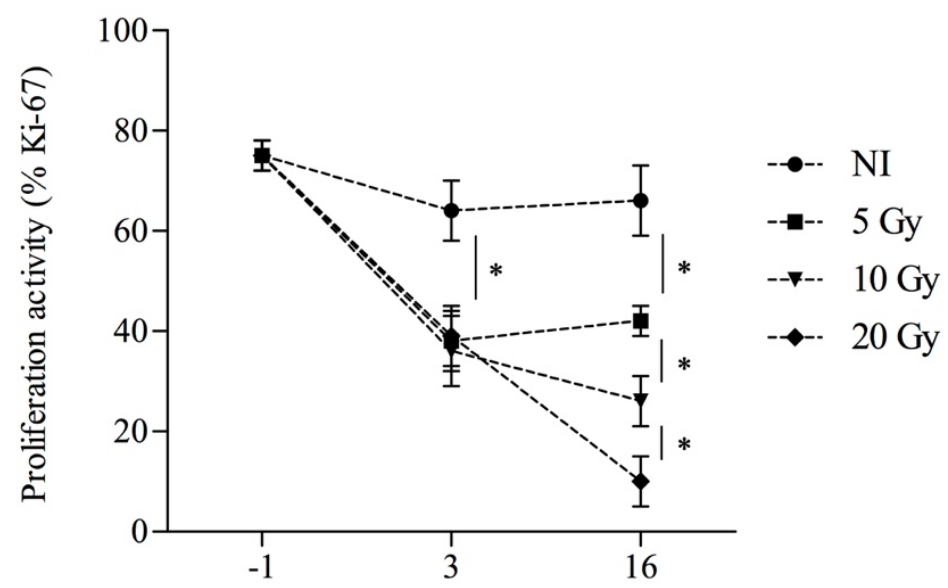

Number of days postirradiation

Figure 6 